\title{
Single-Board Computers na Educação: Uma revisão sistemática da literatura
}

\author{
Single-Board Computers in Education: A systematic literature review \\ Las Computadoras de Tarjeta Única en la Educación: Una revisión sistemática de la literatura
}

Recebido: 24/05/2021 | Revisado: 30/05/2021 | Aceito: 01/06/2021 | Publicado: 17/06/2021

\author{
José Vieira da Costa Neto \\ ORCID: https://orcid.org/0000-0002-1579-8882 \\ Universidade Federal do Ceará, Brasil \\ E-mail: josevieira@ufc.br \\ Edgar Marçal de Barros Filho \\ ORCID: https://orcid.org/0000-0001-5037-2724 \\ Universidade Federal do Ceará, Brasil \\ E-mail: edgar@virtual.ufc.br \\ José Rogério Santana \\ ORCID: https://orcid.org/0000-0002-8327-5864 \\ Universidade Federal do Ceará, Brasil \\ E-mail: rogesantana@ufc.br
}

\begin{abstract}
Resumo
Os Single-Board Computers (SBCs) são computadores de placa única, que possuem baixo custo, consumo de energia reduzido e tamanho exíguo. Podem ser aplicados em várias situações, por exemplo em projetos acadêmicos e data centers. O presente artigo apresenta o resultado de uma Revisão Sistemática da Literatura (RSL) que procurou identificar, em níveis nacional e internacional, a contribuição dos SBCs como ferramentas para apoio ao ensino, em artigos publicados, em periódicos e conferências, entre os anos de 2017 e 2021. Foram definidas uma questão central e três específicas para nortear a pesquisa, juntamente com a aplicação dos critérios de inclusão e exclusão, sendo possível realizar a seleção e extração dos dados. Os resultados alcançados evidenciam que o Raspberry Pi é o SBC que vem sendo mais utilizado, principalmente nas áreas de ensino de ciências, tecnologias, engenharias e matemática, por meio das seguintes abordagens principais: gamificação, cursos, laboratórios virtuais e plataformas online.
\end{abstract}

Palavras-chave: Single-board computers; Raspberry Pi; Educação; Ensino.

\begin{abstract}
Single-Board Computers (SBCs) are single-board computers, which have low cost, reduced energy consumption and small size. They can be applied in various situations, for example in academic projects and data centers. This article presents the result of a Systematic Literature Review (RSL) that sought to identify, at national and international levels, the contribution of SBCs as tools to support teaching, in published articles, in journals and conferences, between the years 2017 and 2021. A central question and three specific ones were defined to guide the research, together with the application of the inclusion and exclusion criteria, making it possible to carry out the selection and extraction of the data. The results achieved show that the Raspberry Pi is the SBC that has been most used, mainly in the areas of science, technology, engineering and mathematics teaching, through the following main approaches: gamification, courses, virtual laboratories and online platforms.
\end{abstract}

Keywords: Single-board computers; Raspberry Pi; Education; Teaching.

\section{Resumen}

Las computadoras de placa única (SBC) son computadoras de placa única, que tienen un bajo costo, un consumo de energía reducido y un tamaño pequeño. Se pueden aplicar en diversas situaciones, por ejemplo, en proyectos académicos y centros de datos. Este artículo presenta el resultado de una Revisión de Literatura Sistemática (RSL) que buscó identificar, a nivel nacional e internacional, el aporte de las SBC como herramientas de apoyo a la docencia, en artículos publicados, en revistas y congresos, entre los años 2017 y 2021. Se definieron una pregunta central y tres específicas para orientar la investigación, junto con la aplicación de los criterios de inclusión y exclusión, permitiendo realizar la selección y extracción de los datos. Los resultados obtenidos muestran que la Raspberry Pi es el SBC que más se ha utilizado, principalmente en las áreas de ciencia, tecnología, ingeniería y enseñanza de las matemáticas, a través de los siguientes enfoques principales: gamificación, cursos, laboratorios virtuales y plataformas online.

Palabras clave: Computadoras de placa única; Raspberry Pi; Educación; Enseñando. 


\section{Introdução}

Atualmente, com o forte avanço tecnológico que o mundo enfrenta, o uso de Tecnologias Digitais da Informação e Comunicação (TDIC) como ferramentas para diversas áreas de ensino aumentou significativamente. Isso permitiu que os alunos acessassem de modo considerável a informação, de forma que sejam preparados para a utilização de diversos recursos tecnológicos existentes.

O uso dos computadores de placa única (do inglês, Single-Board Computers), segundo Basford (2020), tem se tornado popular desde 2012, com mais de 19 milhões de vendas do Raspberry Pi. Eles podem ser utilizados em projetos acadêmicos, data centers (centros de processamento de dados) e clusters portáteis, devido ao seu baixo consumo de energia, baixo aquecimento e seu tamanho reduzido, de forma que possibilita ainda que sejam acoplados vários periféricos (Basford, 2020).

Devido ao seu baixo custo, Dovicchi (2015) destaca a aplicabilidade do Raspberry Pi em disciplinas dos cursos de graduação de computação e engenharias. Os alunos desses cursos podem utilizar suas próprias placas e desenvolver suas atividades práticas com máquinas reais, pois desobriga o uso de simuladores de ambientes computacionais.

Diversos estudos apontam que há um aumento no uso de tecnologias móveis, conforme Marçal, Kubrusly \& Silva (2018). A respeito desse uso na educação, Martins (2018) apresenta alguns benefícios, como por exemplo, a ampliação das experiências de aprendizagem dentro e fora da sala de aula, a facilitação na comunicação, o uso de áudio e vídeo, entre outros. O potencial desses dispositivos na educação surge a partir da utilização das tecnologias móveis como parte de um modelo de aprendizagem integrado, caracterizado pelo uso das ferramentas sem fio de forma transparente e com alto grau de mobilidade (Marçal, Andrade \& Rios, 2005). Contudo, o uso em específico de SBCs se transformou em objeto de pesquisa, devido a sua baixa exploração como metodologia no ensino.

Dessa forma, percebe-se que as pesquisas que acompanham o crescimento no uso de SBCs na Educação, se tornam essenciais. Elas trazem o conhecimento, a compreensão e a aplicação dessas tecnologias como ferramentas para apoio de diversas áreas de ensino, como ensino de ciências naturais, matemática, idiomas, artes, programação, robótica educacional, entre outras.

O objetivo deste artigo é identificar se os SBCs podem contribuir como ferramentas para apoio ao ensino, através de uma Revisão Sistemática da Literatura. Procura-se identificar quais os equipamentos estão sendo utilizados, em quais áreas de ensino são aplicados, e quais as metodologias que estão sendo abordadas. A RSL foi feita com base em publicações de artigos nacionais e internacionais, realizados entre os anos de 2017 e 2021.

\section{Metodologia}

Para Kitchenham (2007) a Revisão Sistemática da Literatura deve ser elaborada por meio de uma série de etapas, dessa forma, permite a sua identificação, avaliação, interpretação e a sistematização de todo o processo de revisão da literatura. A primeira etapa que deve ser realizada é o objetivo da pesquisa, com a definição das questões norteadoras, o campo, junto das bibliotecas determinadas e o período. Logo em seguida, é feita a busca de trabalhos nas bibliotecas, obtém-se, então, os primeiros resultados que deverão ser filtrados através de aplicação dos critérios de inclusão e exclusão. A próxima etapa é a avaliação das publicações com base em alcançar o objetivo da pesquisa. Na etapa final, as publicações que permaneceram deverão ser analisadas e tabuladas de acordo com as questões norteadoras da pesquisa.

\subsection{Questões da Pesquisa}

Acerca das questões da pesquisa, Kitchenham (2007) define como uma das partes fundamentais da Revisão Sistemática da Literatura, que possibilita ao pesquisador buscar trabalhos importantes e relevantes. Contudo, foi definida uma questão central para nortear o objetivo deste trabalho: Os Single-Board Computers podem contribuir como ferramentas para o apoio ao ensino? Foram definidas as seguintes questões da pesquisa na Tabela 1, para alcançar este objetivo. 
Tabela 1 - Questões da Pesquisa.

\begin{tabular}{ll}
\hline Questões & Descrição \\
\hline QP1 & Quais os tipos de SBCs que estão sendo utilizados? \\
QP2 & Em quais áreas de ensino estão sendo aplicados os SBCs? \\
QP3 & Quais metodologias ou estratégias de ensino estão fazendo uso dos SBCs? \\
\hline
\end{tabular}

\subsection{Estratégia de Busca}

Foi realizada uma busca por trabalhos publicados nos últimos cinco anos, entre 2017 e 2021, em que fossem utilizados termos que envolvam "Metodologia", "Ensino" e "Single-Board Computers", nos títulos, resumos ou palavras-chave dos artigos publicados em periódicos ou conferências nas seguintes bibliotecas digitais: Portal de Periódicos da Capes, IEEE Xplore e ScienceDirect, de acordo com a string de busca abaixo.

Os termos utilizados foram organizados na seguinte string de busca: (("Single-Board Computer" OR "Raspberry Pi" OR “Orange Pi”) AND (“Metodologias” OR “Methodologies”) AND (“Ensino” OR “Teaching”)).

Antes da definição da string acima, foram testadas outras formas com termos similares, porém estas strings não puderam ser aplicadas nas três bibliotecas definidas. Uma biblioteca gerou os resultados esperados, outra não conseguiu trazer os resultados mínimos necessários para aplicar os critérios de inclusão e exclusão e outra não conseguiu processar a string criada.

Decidiu-se então utilizar o nome de duas SBCs (Raspberry Pi e Orange Pi) como alternativa para o termo "SingleBoard Computers", e utilizar os termos em inglês "Methodologies" e "Teaching" para "Metodologias" e "Ensino" consecutivamente, haja vista que foram definidos os idiomas português e inglês, por não terem sido encontradas muitas pesquisas somente no idioma português.

Consegue-se, assim, definir e utilizar a mesma string de busca para todas as bibliotecas digitais, de forma menos restritiva na busca, sendo possível obter resultados interessantes, onde foram encontradas, inicialmente, um total de 239 publicações, Gráfico 1. Isto possibilitou que na etapa seguinte pudessem ser aplicados os critérios de inclusão e de exclusão corretamente e critérios de avaliação da qualidade dos trabalhos a partir da análise completa.

Gráfico 1 - Porcentagem de publicações.

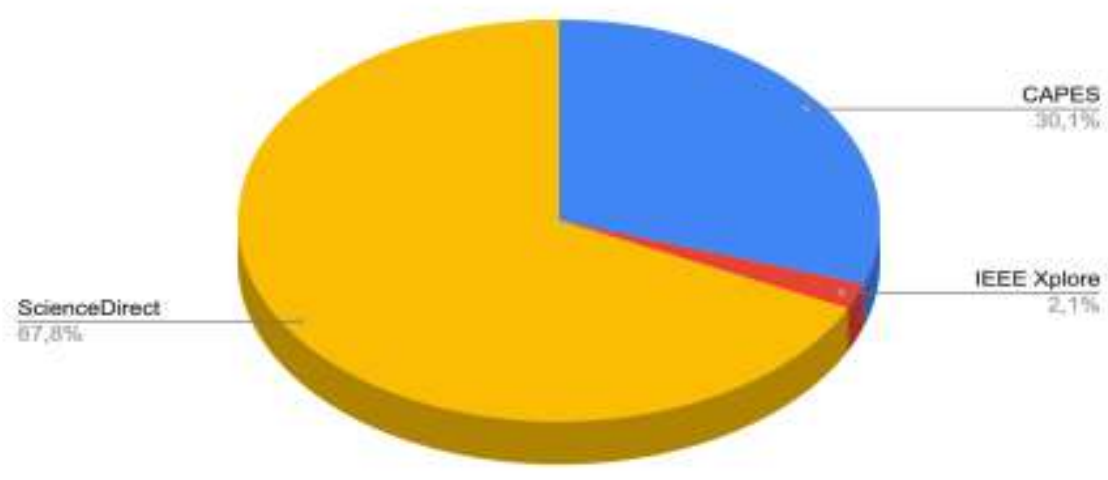

Fonte: Autores. 


\subsection{Condução com a Aplicação dos Critérios de Inclusão e Exclusão}

Kitchenham (2007) define que a aplicação dos critérios de inclusão e exclusão permite o alcance dos objetivos da pesquisa, a selecionar trabalhos equivalentes. Adiante, os critérios de inclusão e exclusão foram aplicados, conforme a Tabela 2, foram efetuadas leituras das publicações nos títulos, resumos e palavras-chave, onde restaram 34 artigos, que tiveram seus textos analisados na íntegra para a avaliação da qualidade.

Tabela 2 - Critérios de Inclusão e Exclusão.

\begin{tabular}{ll}
\hline Critérios de Inclusão $(\mathrm{CI})$ & Critérios de Exclusão (CE) \\
\hline CI1: Utiliza Single-Board Computers & CE1: Trabalhos que não são artigos \\
CI2: Aplicabilidade na área da Educação & CE2: Não está em português ou inglês \\
CI3: Artigos empíricos ou teóricos & CE3: Não utiliza SBC \\
$\begin{array}{l}\text { CI4: Artigos que abordam o processo de ensino } \\
\text { aprendizagem através de SBC }\end{array}$ & $\begin{array}{l}\text { CE4: Artigos sem enfoque no ensino ou na } \\
\text { aprendizagem }\end{array}$ \\
\hline
\end{tabular}

Fonte: Autores.

Realizou-se uma análise completa nos textos dos 34 artigos selecionados, onde aplicou-se os critérios de inclusão e exclusão junto das questões da pesquisa, dessa forma, busca-se, obter uma avaliação de qualidade e adequação das publicações restantes com o objetivo proposto da Revisão Sistemática da Literatura, onde se obteve o resultado final de 17 artigos para extração dos dados, conforme mostra a Figura 1.

Figura 1 - Artigos Resultantes.

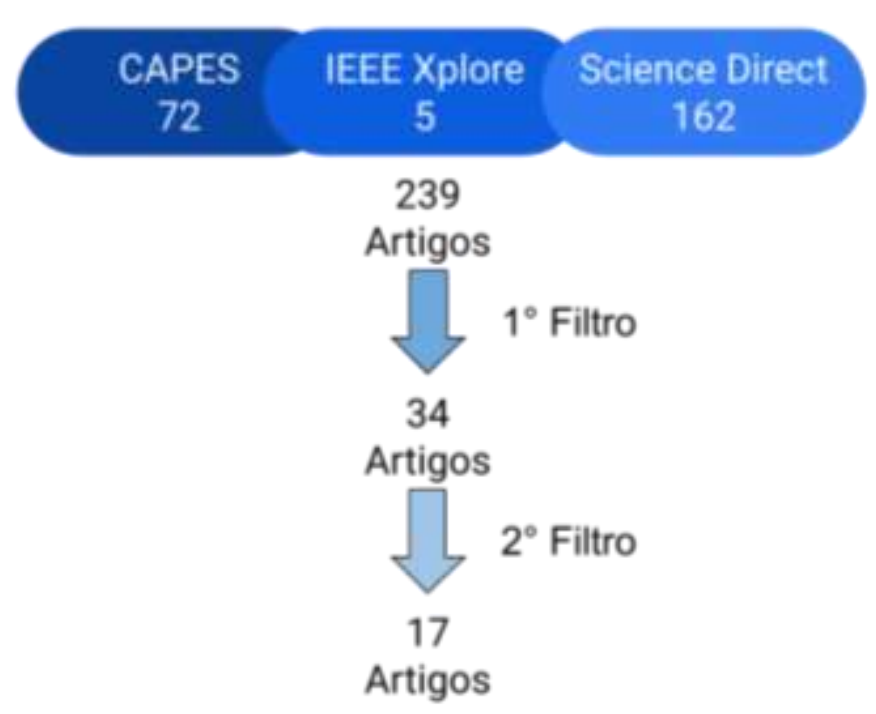

Fonte: Autores.

Durante a etapa de aplicação dos critérios de inclusão e exclusão das publicações, identificou-se alguns artigos de Revisão Sistemática da Literatura de assuntos próximos ao contexto de Single-Board Computers realizados nos últimos cinco anos, como movimento maker, realidade virtual, entre outros. Decidiu-se, então, aplicar as questões da pesquisa, onde nenhuma das publicações atenderam aos requisitos estabelecidos pela RSL.

Ainda na íntegra leitura dos 34 artigos, identificou-se publicações que utilizavam arduino ou outros microcontroladores, e não SBCs para o ensino, assim como também descartou-se artigos que comentam sobre o uso, mas não aplicam na prática ou não 
afirmam a sua possibilidade. E, por fim, trabalhos que não utilizam SBCs como estratégias para o ensino. A seguir apresenta-se os resultados e discussões com as respostas das três questões de pesquisa elaboradas de acordo com cada artigo analisado.

\section{Resultados e Discussão}

A partir da análise dos 17 trabalhos restantes, extraiu-se e catalogou-se os dados com informações referentes à quantidade de artigos em cada biblioteca digital, data de leitura, link da pesquisa, título, respostas das perguntas norteadoras, autores, fonte de cada artigo, ano da publicação, local e outras informações. O Quadro 1 traz uma lista dos trabalhos escolhidos, com o título, o seu ano, e a biblioteca digital encontrada para extração dos dados, junto com suas IDs. Buscou-se, dessa forma, catalogar as referências a fim de facilitar as discussões de cada questão de pesquisa.

Quadro 1 - Lista dos trabalhos escolhidos.

\begin{tabular}{|c|c|c|c|}
\hline ID & Título & Biblioteca Digital & Ano \\
\hline 1 & A novel approach to learning music and piano based on mixed reality and gamification & Periódicos CAPES & 2021 \\
\hline 2 & $\begin{array}{l}\text { AAL Platform with a "De Facto" Standard Communication Interface (TICO): Training } \\
\text { in Home Control in Special Education }\end{array}$ & Periódicos CAPES & 2017 \\
\hline 3 & $\begin{array}{l}\text { Design and Implementation of Web-Based Virtual Mobile Robot Laboratory for } \\
\text { Engineering Education }\end{array}$ & Periódicos CAPES & 2020 \\
\hline 4 & $\begin{array}{l}\text { Design, simulation and testing of a cloud platform for sharing digital fabrication } \\
\text { resources for education }\end{array}$ & Periódicos CAPES & 2019 \\
\hline 5 & $\begin{array}{l}\text { Ecosystem for the deployment and management of virtual laboratories based on the } \\
\text { standard IMS LTI }\end{array}$ & Periódicos CAPES & 2019 \\
\hline 6 & Enhancing EJsS with Extension Plugins & Periódicos CAPES & 2021 \\
\hline 7 & $\begin{array}{l}\text { Introducing Low-Cost Sensors into the Classroom Settings: Improving the Assessment } \\
\text { in Agile Practices with Multimodal Learning Analytics }\end{array}$ & Periódicos CAPES & 2019 \\
\hline 8 & Toward a telepresence robot empowered smart lab & Periódicos CAPES & 2019 \\
\hline 9 & Teaching HPC Systems and Parallel Programming with Small-Scale Clusters & IEEE Xplore & 2018 \\
\hline 10 & $\begin{array}{l}\text { Towards education alternatives to teaching and learning of programming: A course } \\
\text { experience using open hardware tools }\end{array}$ & IEEE Xplore & 2018 \\
\hline 11 & Development of a Design Education Platform for an Interdisciplinary Teaching Concept & ScienceDirect & 2020 \\
\hline 12 & $\begin{array}{l}\text { Design and implementation of a low cost RFID track and trace system in a learning } \\
\text { factory }\end{array}$ & ScienceDirect & 2018 \\
\hline 13 & $\begin{array}{l}\text { Teaching Industrie } 4.0 \text { technologies in a learning factory through problem-based } \\
\text { learning: case study of a semi-automated robotic cell design }\end{array}$ & ScienceDirect & 2020 \\
\hline 14 & On the challenges novice programmers experience in developing IoT systems: A Survey & ScienceDirect & 2019 \\
\hline 15 & An Approach of Training Virtual Environment for Teaching Electro-Pneumatic Systems & ScienceDirect & 2019 \\
\hline 16 & A design of a short course with COTS UAV system for higher education students & ScienceDirect & 2019 \\
\hline 17 & $\begin{array}{l}\text { Autonomous Vehicle Control based on HoloLens Technology and Raspberry Pi } \\
\text { Platform: an Educational Perspective }\end{array}$ & ScienceDirect & 2019 \\
\hline
\end{tabular}




\subsection{QP1 - Quais os tipos de SBCs que estão sendo utilizados?}

A partir desta questão foi possível identificar quais computadores de placa única foram utilizados, Tabela 3, a seguir. Dos 17 artigos listados, 15 utilizam Raspberry Pi, onde os trabalhos [1], [4] e [17] detalham que a versão 3 é utilizada, o trabalho [12] foi mais específico ainda, detalha o uso da versão 3, modelo B e o trabalho [11] relata o uso da versão zero. Já os trabalhos [3] e [9] utilizam outras SBCs, sendo uma BeagleBoard-xM e uma Odroid-XU4 consecutivamente. Os outros 10 trabalhos não possuem detalhes de qual versão da Raspberry $P i$ foi utilizada.

Tabela 3 - Tipos de SBCs.

\begin{tabular}{cl}
\hline Qtd. & Single-Board Computer \\
\hline 10 & Raspberry Pi \\
3 & Raspberry Pi 3 \\
1 & Raspberry Pi 3B \\
1 & Raspberry Pi Zero \\
1 & BeagleBoard-xM \\
1 & Odroid-XU4 \\
\hline & Fonte: Autores.
\end{tabular}

A grande difusão no mercado mundial, a maior compatibilidade de softwares desenvolvidos, o grande número de sistemas operacionais e comunidades envolvidas e o custo equivalente aos outros SBCs podem ter sido os motivos para maior utilização do Raspberry Pi nos trabalhos analisados. A Figura 2 ilustra o uso dos SBCs para apoiar o ensino em um curso de formação de professores (Nuñez-Unda, et al, 2018).

Figura 2 - Formação de professores com Raspberry Pi.

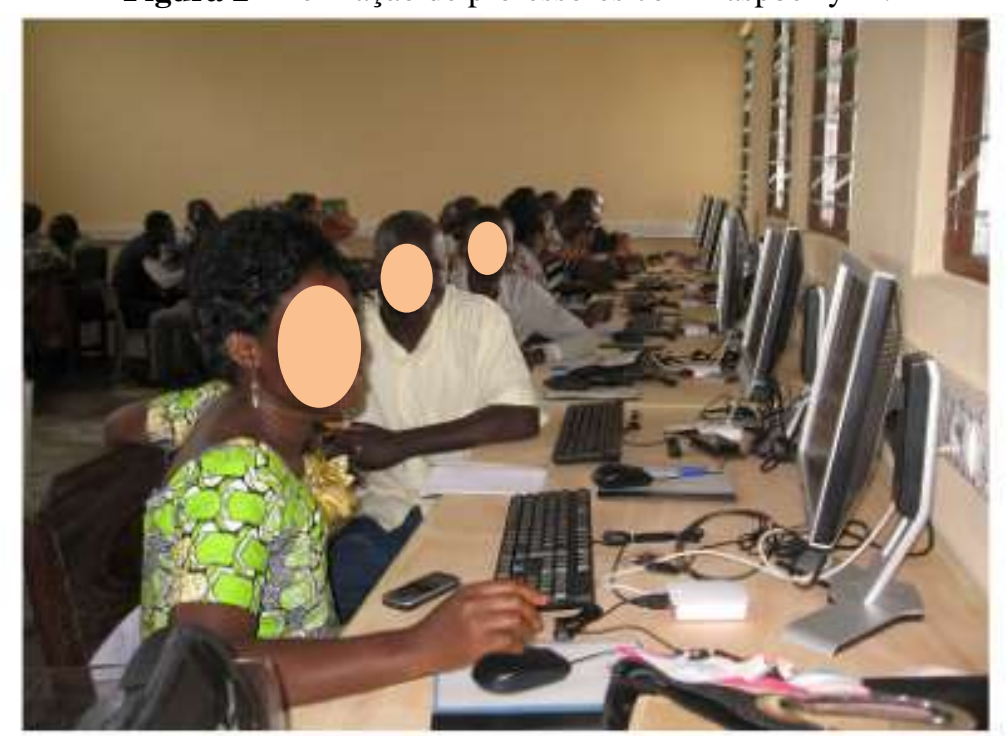

Fonte: Nuñez-Unda, et al. (2018).

\subsection{QP2 - Em quais áreas de ensino estão sendo aplicados os SBCs?}

Nesta questão identificou-se diversas áreas de ensino, divididas em 3 classificações, como mostra o Gráfico 2. A primeira área foi o ensino de música, que contém apenas o trabalho [1], onde é ensinado piano. Outro trabalho selecionado foi o 
[2], que envolve educação especial. Já os 15 trabalhos seguintes abordam áreas de ensino como ciências, tecnologia, engenharia e matemática (STEM - Science, Technology, Engineering and Mathematics).

Os trabalhos [3] e [11] abordam o ensino interdisciplinar em engenharias, os [4] e [6] são mais abrangentes, versam sobre o ensino e aprendizagem de disciplinas de STEM como um todo. Já os trabalhos [5] e [10] tratam do ensino de programação, enquanto o [7] traz o ensino de engenharia de software. $\mathrm{O}$ [8] relata apenas o ensino de ciências, o [9] procura realizar o ensino de sistemas de computação de alta performance (HPC) e programação paralela. Os [12] e [13] abordam acerca do ensino de conceitos e tecnologias da Indústria 4.0. O trabalho [14] refere-se sobre o ensino de internet das coisas (IoT Internet of Things), o [15] retrata o ensino de sistemas eletropneumáticos, já o [16] desenvolve o ensino de drones e o último [17], discorre sobre o ensino de sistemas de controle.

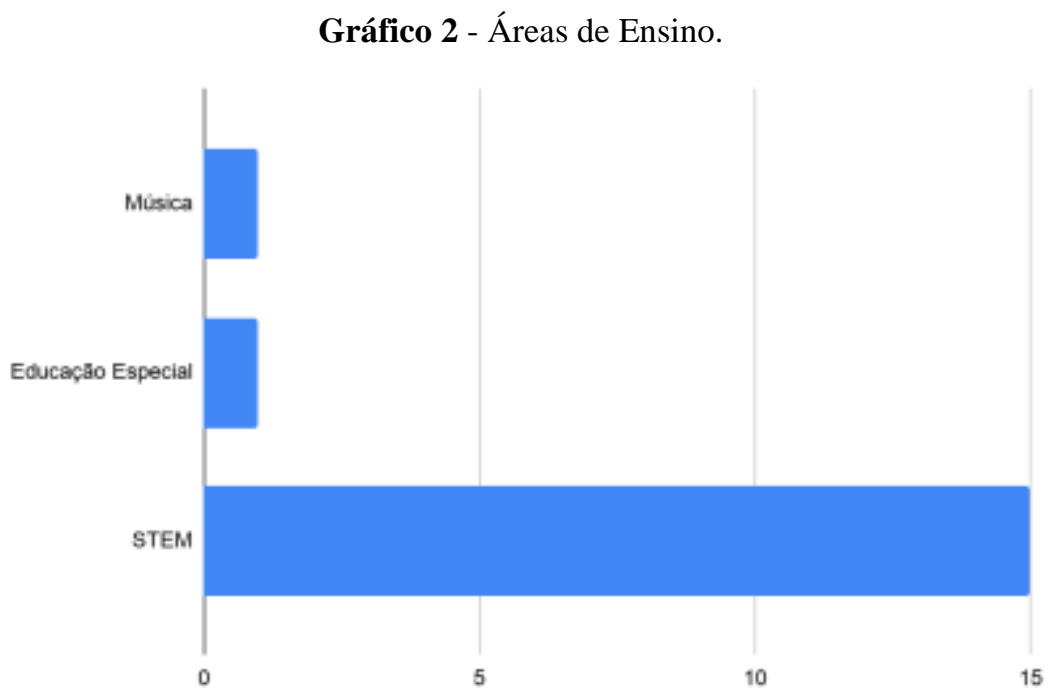

Fonte: Autores.

Áreas de ensino STEM apareceram na grande maioria dos trabalhos selecionados, onde foram identificados principalmente trabalhos que envolvem o ensino de tecnologias e engenharia.

\subsection{QP3 - Quais metodologias ou estratégias de ensino estão fazendo uso dos SBCs?}

Esta questão tem o objetivo de identificar como os SBCs estão sendo utilizados para conduzir as disciplinas ministradas. Portanto, os seus dados foram analisados tanto de forma quantitativa, para categorizar os tipos de metodologias e estratégias utilizadas nas áreas de ensino, conforme Tabela 4, quanto de forma qualitativa, buscando dar atenção maior em cada trabalho, não só categorizando, mas identificando a especificidade de cada abordagem.

Tabela 4 - Metodologias e Estratégias.

\begin{tabular}{llr}
\hline Metodologias/Estratégias & Trabalhos & Qtd \\
\hline Gamificação & {$[1]$} & 1 \\
Cursos & {$[16]$} & 1 \\
Robótica & {$[3],[8],[11],[13],[16],[17]$} & 6 \\
Laboratórios Virtuais & {$[3],[4],[5],[6],[8],[15]$} & 6 \\
Sistemas / Ferramentas / Plataformas / Estruturas & {$[1],[2],[7],[9],[10],[11],[12],[13],[14],[17]$} & 10 \\
\hline
\end{tabular}


No trabalho [1], é abordado o uso de gamificação e realidade híbrida. A estrutura é composta através de um Raspberry Pi 3, que é conectado a um teclado por meio de um adaptador USB-MIDI. É utilizado o software amidi e um script em python para enviar os dados para o Microsoft Hololens (capacete holográfico), que permite que o aluno visualize a tela do jogo e avance os níveis de aprendizado.

Procurando criar uma plataforma para estudar adaptações e estratégias de formação que potenciem as oportunidades de autonomia dos seus alunos, o trabalho [2] utiliza um Raspberry Pi como sistema de controle para gerenciar luzes, televisão, motores de janelas persianas, por meio de portas USB, GPIO, conexões infravermelho, wifi, etc. O que possibilita um treinamento em habilidades alternativas de comunicação e controle doméstico em Educação Especial.

Consoante os trabalhos [1] e [2], os trabalhos [7], [9], [10], [11], [12], [13], [14] e [17] fazem o uso de sistemas, ferramentas, plataformas e estruturas como metodologias para o ensino de STEM. O trabalho [7] utiliza um Raspberry Pi com uma placa de expansão de 4 microfones (ReSpeaker), emprega um software MMLA para gravar e armazenar as intervenções de fala dos alunos. Dessa forma o trabalho consegue identificar as oportunidades em que falavam, bem como a duração de cada intervenção realizada para Análise de Aprendizagem Multimodal por meio de monitoramento de colaboração automatizado.

No trabalho [9] são utilizadas placas Odroid-XU4 para um cluster de SBC de pequena escala e o software OpenMP para ensino de programação paralela. O [10] explora ferramentas de hardware abertas e componentes industriais, utilizando Raspberry Pi e linguagem Python para programar e manusear os sensores e atuadores. Uma plataforma de design em educação, por meio de um sistema robótico físico, foi desenvolvida no trabalho [11], que utilizou um Raspberry Pi Zero como unidade de controle e comunica com a interface do usuário, controlada por um controle de Xbox One.

Um sistema RFID de rastreamento de baixo custo é criado no trabalho [12], através de um Raspberry Pi 3B. A estrutura executa um servidor LAMP (Linux, Apache, MySQL e PHP) que recebe, armazena e disponibiliza os dados RFID para serem tratados pelo software de BI (Business Inteligence) InfoCaptor. No trabalho [13] um projeto de uma célula robótica semiautomática usa um Raspberry Pi para iniciar e parar remotamente o robô e transmitir um vídeo da operação da célula robótica para o navegador de quem estiver no controle.

No trabalho [14], uma arquitetura de sistemas IoT genérica em uma pesquisa com desenvolvedores novatos utiliza Raspberry Pi como um gateway, que reúne os dados dos sensores e interage com os atuadores, ambos subsistemas caracterizados. O trabalho [17] aborda experimentos com localização, mapeamento e controle de veículos autônomos e utiliza um Raspberry Pi 3 para controlar os motores servo e DC. Este envia os dados para um banco de dados SQL e a aplicação web Node-RED que em seguida encaminha para o HoloLens.

Sobre os trabalhos que abordam robótica, temos os [11], [13] e [17] citados anteriormente e o trabalho [3], que utiliza um BeagleBoard-xM que controla o robô e todos seus componentes, por meio de um servidor web LightHTTPd o que propicia um ambiente de laboratório virtual e remoto baseado na web. Semelhante a ele temos [8], que utiliza um Raspberry Pi junto com uma webcam $H D$ e executa um servidor de streaming de vídeo (WebRTC). Este pode também ser utilizado para adquirir dados de sensores no laboratório e transmitir para o usuário do laboratório remoto inteligente habilitado por robô de telepresença. Para promover um curso de curta duração com um sistema de veículo aéreo não tripulado comercial pronto para uso (COTS UAV), [16] faz uso de um Raspberry Pi com uma câmera e um sensor de deteç̧ão de luz e alcance (LiDAR - Light Detection And Ranging), integrado com um controlador de voo Pixhawk.

Também foram categorizados trabalhos que abordaram o uso de SBCs como ferramenta para laboratórios virtuais, como os [3] e [8], citado anteriormente e os trabalhos [4], [5], [6] e [15]. No trabalho [4] utiliza-se um Raspberry Pi 3 junto das máquinas de fabricação do Fab Lab, o que possibilita a esses equipamentos enviarem as informações em tempo real pela internet, com tecnologias de nuvem para oferecer acesso remoto a elas, dessa maneira o ensino e aprendizagem de disciplinas de STEM se torna mais atraente. 
Outro trabalho acerca de laboratórios é o [5]. Nele é usado um Raspberry Pi como um servidor, que compila o código que o usuário insere na sua interface, executa comandos na placa arduino e realiza também uma transmissão de vídeo da forma que permita ao usuário observar o que acontece no laboratório. Já o [6] versa sobre o software open source EJsS (Easy JavaJavascript Simulations) para um laboratório remoto de ensino de ciências, matemática e física e recomenda a aplicação de um Raspberry Pi para sua implantação e a utilização de um módulo wiringPi com as suas portas GPIO e o pyserial para comunicar com dispositivos Serial/USB.

Por fim, temos o [15], onde dispõe-se de um Raspberry Pi junto do software mosquitto, que utiliza protocolo MQTT, para comunicar e enviar instruções de controle com o sistema eletropneumático real FESTO e interpretá-las no ambiente desenvolvido de realidade virtual, através do Unity Pro. Dessa forma, é realizada a criação de um ambiente de treinamento prático para a simulação e controle de um processo pneumático.

Ao realizar análise das metodologias, métodos e estratégias que os SBCs foram aplicados nas áreas de ensino, percebeuse que grande parte dos trabalhos abordam seu uso para criar ferramentas ou plataformas que proporcionem a criação de laboratórios virtuais e remotos. Dessa maneira é possível que integrem componentes de robótica e substituam, de forma eficiente, microcontroladores como o arduino, devido principalmente à possibilidade que eles têm de execução de sistemas operacionais baseados em Linux e utilização de diversos serviços de rede.

\section{Considerações Finais}

Este artigo realizou uma Revisão Sistemática da Literatura que identificou a contribuição dos Single-Board Computers como ferramentas para apoio ao ensino, por meio da análise de artigos publicados entre os anos de 2017 e 2021 . A partir de um total de 239 trabalhos encontrados, 34 foram avaliados com os critérios de inclusão e exclusão e aplicação das questões da pesquisa, que resultaram apenas 17 trabalhos que foram discutidos após a sua análise na íntegra.

A análise dos artigos demonstrou que os SBCs são ferramentas que melhoram o ensino em diversas áreas. O Raspberry $P i$ aparece como o SBC mais utilizado nos trabalhos escolhidos. As áreas de ensino de tecnologia e engenharia são as mais destacadas. As principais metodologias utilizadas foram gamificação, cursos, laboratórios virtuais e uso de plataformas.

Como trabalho futuro, recomenda-se novas pesquisas bibliográficas, que procurem abordar o uso de SBCs em outras áreas de ensino fundamental e médio como as artes, as linguagens, os jogos educacionais, entre outros. Aconselha-se que haja direcionamento de pesquisas também no ensino superior em cursos mais voltados para formação docente como pedagogia e licenciaturas.

\section{Referências}

Alvarez, L., Ayguade, E. \& Mantovani, F. (2018). Teaching hpc systems and parallel programming with small-scale clusters. In: 2018 IEEE/ACM Workshop on Education for High-Performance Computing (EduHPC). IEEE. p. 1-10.

Ariza, J. Á. (2018). Towards education alternatives to teaching and learning of programming: A course experience using open hardware tools. In: 2018 IEEE Frontiers in Education Conference (FIE). IEEE. p. 1-8.

Barros Filho, E. M., Kubrusly, M. \& Silva, C., L. O. (2018). Avaliando Aplicações Móveis para o Ensino em Saúde: Uma Revisão Sistemática. Tecnologia Educacional [On Line], Rio De Janeiro, N. 221, P. 40-50, Issn: 0102-5503.

Basford, P. J. et al. (2020). Performance analysis of single board computer clusters. Future Generation Computer Systems. 102, $278-91$.

Chacon, J. et al. (2021). Enhancing EJsS with Extension Plugins. Electronics, v. 10, n. 3, p. 242.

Cornetta, G. et al. (2019). Design, simulation and testing of a cloud platform for sharing digital fabrication resources for education. Journal of Cloud Computing, v. 8 , n. 1, p. 1-22.

Cornide-Reyes, H. et al. (2019). Introducing low-cost sensors into the classroom settings: Improving the assessment in agile practices with multimodal learning analytics. Sensors, v. 19, n. 15, p. 3291.

Corno, F., De Russis, L. \& Sáenz, J. P. (2019). On the challenges novice programmers experience in developing IoT systems: A survey. Journal of Systems and Software, v. 157, p. 110389. 
Dovicchi, J. (2015). O uso do Raspberry Pi em laboratórios de aulas práticas: uma Contribuição ao Ensino de Ciências da Computação. INE/UFSC.

Garcia, C. A., Caiza, G., Naranjo, J. E., Ortiz, A., \& Garcia, M. V. (2019). An approach of training virtual environment for teaching electro-pneumatic systems. IFAC-PapersOnLine, 52(9), 278-284.

González-Amarillo, Á. M. (2019). Ecosystem for the deployment and management of virtual laboratories based on the standard IMS LTI. Revista Facultad de Ingeniería, 28(53), 79-99.

Guillomía San Bartolomé, M. A., Falco Boudet, J. L., Artigas Maestre, J. I., \& Sanchez Agustin, A. (2017). AAL platform with a “de facto” standard communication interface (TICO): Training in home control in special education. Sensors, 17(10), 2320.

Heyden, E., Küchenhof, J., Greve, E., \& Krause, D. (2020). Development of a Design Education Platform for an Interdisciplinary Teaching Concept. Procedia CIRP, 91, 553-558.

Hong, J. H., Shin, H. S., \& Tsourdos, A. (2019). A design of a short course with COTS UAV system for higher education students. IFAC-PapersOnLine, 52(12), 466-471.

Kitchenham, B., \& Charters, S. (2007). Guidelines for performing systematic literature reviews in software engineering.

Louw, L., \& Walker, M. (2018). Design and implementation of a low cost RFID track and trace system in a learning factory. Procedia Manufacturing, 23, 255260 .

Louw, L., \& Deacon, Q. (2020). Teaching Industrie 4.0 technologies in a learning factory through problem-based learning: case study of a semi-automated robotic cell design. Procedia Manufacturing, 45, 265-270.

Marçal, E., Andrade, R., \& Rios, R. (2005). Aprendizagem utilizando dispositivos móveis com sistemas de realidade virtual. RENOTE-Revista Novas Tecnologias na Educação, 3(1).

Martins, E. R., Geraldes, W. B., Afonseca, U. R., \& Gouveia, L. M. B. (2018). Tecnologias Móveis em Contexto Educativo: uma Revisão Sistemática da Literatura. RENOTE, 16(1).

Moezzi, R., Krcmarik, D., Bahri, H., \& Hlava, J. (2019). Autonomous vehicle control based on HoloLens technology and raspberry pi platform: An educational perspective. IFAC-PapersOnLine, 52(27), 80-85.

Molero, D., Schez-Sobrino, S., Vallejo, D., Glez-Morcillo, C., \& Albusac, J. (2021). A novel approach to learning music and piano based on mixed reality and gamification. Multimedia Tools and Applications, 80(1), 165-186.

Nuñez-Unda, A., Vera, A., Haz, L., Pinos, V., Zurita, R., \& Medina, S. (2018). The raspberry Pi as a computer substitute at elementary schools in developing countries: a pilot experiment in ecuador. In MATEC Web of Conferences (Vol. 210, p. 04023). EDP Sciences.

Solak, S., Yakut, Ö., \& Dogru Bolat, E. (2020). Design and Implementation of Web-Based Virtual Mobile Robot Laboratory for Engineering Education. Symmetry, 12(6), 906.

Tan, Q., Denojean-Mairet, M., Wang, H., Zhang, X., Pivot, F. C., \& Treu, R. (2019). Toward a telepresence robot empowered smart lab. Smart Learning Environments, 6(1), 1-19. 